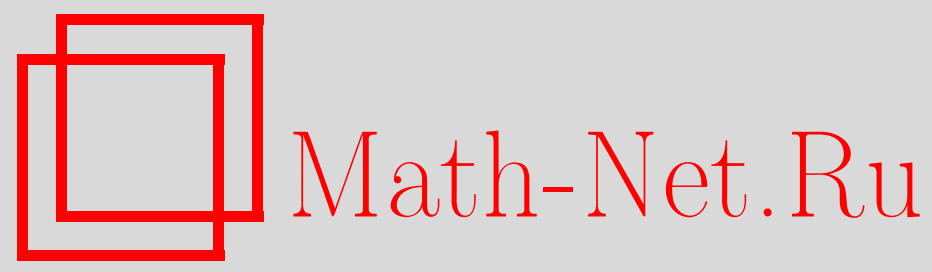

С. С. Рышков, Бесконечные грани совершенного полиэдра Вороного, Матем. заметки, 2004, том 75, выпуск 2, 261268

DOI: https://doi.org/10.4213/mzm32

Использование Общероссийского математического портала Math-Net.Ru подразумевает, что вы прочитали и согласны с пользовательским соглашением http://www. mathnet.ru/rus/agreement

Параметры загрузки:

IP : 54.172.240.79

26 апреля 2023 г., $17: 48: 26$

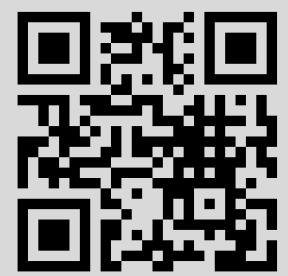




\title{
БЕСКОНЕЧНЫЕ ГРАНИ СОВЕРШЕННОГО ПОЛИЭДРА ВОРОНОГО
}

\author{
С. С. Рышков
}

В статье изучаются бесконечные грани совершенного полиэдра Вороного. Результат оказывается существенно различным в зависимости от того, рассматривать ли совершенный полиэдр Вороного как замкнутую или как незамкнутую выпуклую оболочку множества точек Вороного (см. теорему 1 из п. 5 и теорему 2 из п. 7).

Библиографоял: 2 названия.

Автор посвящает эту статью своему дорогому другу профессору Петеру Манфреду Груберу к его 60-летию.

1. Рассматривается (см., например, [1]) евклидово пространство $\mathbf{E}^{N}=\mathbb{E}(n)$ размерности $N=n(n+2) / 2$, где $n$ - натуральное число. Пространство $\mathbf{E}^{N}$ считается заданным ортонормированной системой координат

$$
\left(v_{11}, v_{22}, \ldots, v_{n, n}, v_{12}, \ldots, v_{n-1, n}\right)
$$

с началом в точке $\mathbf{0 .}$

Каждой точке $f \in \mathbf{E}^{N}$, имеющей координаты

$$
\left(a_{11}, a_{22}, \ldots, a_{n, n}, a_{12}, \ldots, a_{n-1, n}\right),
$$

ставится в соответствие квадратичная форма

$$
f=f(\bar{x})=\sum_{i, j=1}^{n} a_{i j} x_{i} x_{j}=\bar{x}^{T} A \bar{x}
$$

где $a_{i j}=a_{j i}$ - действительные числа, через $A$ обозначена симметрическая матрища чисел $a_{i j}$, через $\bar{x}$ - столбец переменных $x_{1}, x_{2}, \ldots, x_{n}$ и через $T$ обозначена операция транспонирования.

Наоборот, каждой квадратичной форме вида (2) ставится в соответствие точка $f \in$ $\mathbf{E}^{N}$, имеющая координаты (1).

Множество точек $f \in \mathbf{E}^{N}$, соответствующих положительно определенным квадратичным формам вида (2) (далее ПК $\Phi$ ), образует открытьй вьпукльй конус $\mathbb{K}(n) \subset$ $\mathbb{E}(n)$ - конус положительности ранга $n$. Граница $\partial \mathbb{K}(n) \subset \mathbb{E}(n)$ состоит из тех и

Работа поддержана Международным институтом математической физики (ESI), Вена, Австрия, а также Российским фондом фундаментальньг исследований, гранты №№ 97-01-266 и 00-0100619 . 
только тех точек пространства $\mathbb{E}(n)$, которые отвечают неотрицательно определенным квадратичным формам вида (2) ранга меньшего, чем $n$ (неотрииательным, положительно полуопределенным формам), и нулевой форме. Рассматривается также конус $\overline{\mathbb{K}}(n)=\partial \mathbb{K}(n) \cup \mathbb{K}(n)$.

2. Точкой Вороного назьвается каждая точка пространства $\mathbb{E}(n)$, отвечающая квадратичной форме вида $\left(q_{1} x_{1}+q_{2} x_{2}+\cdots+q_{n} x_{n}\right)^{2}$, где $q_{1}, q_{2}, \ldots, q_{n}-$ нижние координаты точки Вороного - целые, невсе равныенулю, числа. Примитивной точкой Вороного назьвается такая точка Вороного, у которой числа $q_{1}, q_{2}, \ldots, q_{n}$ не имеют общего делителя. Очевидно, что все точки Вороного лежат в границе $\partial \mathbb{K}(q)$, конуса $\mathbb{K}(n)$.

ОПРЕДЕЛЕНИЕ 1. Замкнутым (совершенным) полиэдром Вороного (ранга $n$ ) назьвается замкнутая вьпуклая оболочка $\Pi^{+}(n) \subset \overline{\mathbb{K}}(n)$ множества всех точек Вороного.

ОПРЕДЕЛЕНИЕ 2. Незамкнутым (совершенным) полиәдром Вороного (ранга $n$ ) назьвается незамкнутая вьпуклая оболочка $\Pi^{-}(n) \subset \overline{\mathbb{K}}(n)$ множества всех точек Вороного.

Если не важно, какая рассматривается выпуклая оболочка множества всех точек Вороного, замкнутая или нет, то говорят [1] просто о (совершенном) полиэдре Вороного $\Pi(n)$ (ранга $n)$.

Часто в определении полиэдра Вороного рассматривается вьпуклая оболочка множества не всех точек Вороного, а только примитивных; это не корректно при $n=1$ и, тем самым, нарушает дуальность совершенных полиэдров $\Pi^{-}(n)$ и $M(n)$. Однако при рассмотрении лишь конечных граней полиэдра Вороного этой некорректностью, хотя она и затрудняет доказательства, можно пренебречь.

3. В пространстве $\mathbb{E}(n)$ рассматриваются плоскости с уравнениями вида

$$
\sum_{i, j=1}^{n} a_{i j} v_{i j}=C
$$

Если фиксировать значение $C \neq 0$, то каждой квадратичной форме вида $(2)$, не равной тождественно нулю, ставится в соответствие единственная плоскость вида (3) и наоборот. Рассматриваемые плоскости классифицируются как әллиптические, параболические и гиперболические плоскости в зависимости от того, является ли форма $f$ определенной, полуопределенной или неопределенной.

На эллиптических плоскостях могут лежать только конечные грани полиэдра Вороного. На гиперболических плоскостях, а также на параболических плоскостях вида (3) при $C \neq 0$ не лежит никаких граней полиэдра Вороного, см. [1]. (Гранью выпуклого множества называется его пересечение с произвольной опорной к нему плоскостью. Например, каждая точка границы замкнутого метрического шара есть его грань. Открытый метрический шар граней не имеет.)

Итак, нас будут интересовать лишь параболические плоскости $\mathbb{P}_{f}^{N-1}$ вида (3), в основном, при $C=0$; при этом мы будем предполагать, что форма $f$ полуопределена положительно.

4. Наряду с пространством $\mathbf{E}^{N}$ мы будем рассматривать пространство (переменных формы) $\mathbf{E}^{n}$, заданное ортонормированной системой координат $\left(x_{1}, x_{2}, \ldots, x_{n}\right)$. Через $\mathbf{E}^{q}$ 
мы будем обозначать координатную плоскость первых $q$ координат пространства $\mathbf{E}^{n}$, a через $\mathbb{E}(q)$ - пространство квадратичных форм от переменных $\left(x_{1}, x_{2}, \ldots, x_{q}\right)$. Соответствующим образом определяются и множества $\mathbb{K}(q), \partial \mathbb{K}(q), \overline{\mathbb{K}}(q)$.

Уравнение $f(\bar{x})=0$, где $f(\bar{x})$ - положительно полуопределенная форма ранга $p>0$ $(\operatorname{rg} f(\bar{x})=p)$, задает в пространстве $\mathbf{E}^{n}$ плоскость $\mathscr{P} q$, где, как и всюду далее, $p+q=n$. (Например, плоскость $\mathbf{E}^{q}$ задается уравнением $x_{q+1}^{2}+x_{q+2}^{2}+\cdots+x_{n}^{2}=0$.)

В случае, когда плоскость $\mathscr{P}^{q}$ пересекается с решеткой $\mathbb{Z}^{n} \subset \mathbf{E}^{n}$ по подрешетке ранга $q$, плоскость $\mathscr{P} q$ назьвается вполне рациональной. (Например, плоскость $\mathbf{E}^{q}$ вполне рациональна.)

В случае, когда плоскость $\mathscr{P}^{q}$ пересекается с решеткой $\mathbb{Z}^{n} \subset \mathbf{E}^{n}$ лишь по точке $(0,0, \ldots, 0)$, плоскость $\mathscr{P}^{q}$ называется вполне иррациональной. (Промежуточные случаи специальных названий не имеют.)

5. ЛЕМмА 1. Пусть плоскость $\mathbb{P}_{f}^{N-1}$ задана равенством

$$
\sum_{i, j=1}^{n} a_{i j} v_{i j}=0
$$

где $n>\operatorname{rg} f(\bar{x})=p>0$. Тогда пересечение плоскости $\mathbb{P}_{f}^{N-1}$ с конусом $\overline{\mathbb{K}}(n)$ аффинно әквивалентно конусу $\overline{\mathbb{K}}(q)$.

ДокАЗАТЕЛЬСтво. Приведем форму $f(\bar{x})$ к следующему нормальному виду:

$$
f^{\prime}(\bar{x})=x_{q+1}^{2}+x_{q+2}^{2}+\cdots+x_{n}^{2}
$$

Это приведение повлечет за собой некоторое аффинное преобразование, переводящее плоскость $\mathbb{P}_{f}^{N-1}$ в плоскость $\mathbb{P}_{f^{\prime}}^{N-1}$ с уравнением $v_{q+1, q+1}+v_{q+2, q+2}+\cdots+v_{n, n}=0$, а конус $\overline{\mathbb{K}}(n)$ в себя. Заметим, что при этом преобразовании полиэдр Вороного не сохраняется.

Рассмотрим, какие точки конуса $\overline{\mathbb{K}}(n)$ принадлежат плоскости $\mathbb{P}_{f^{\prime}}^{N-1}$. Пусть

$$
g(\bar{x})=g\left(a_{11}, a_{22}, \ldots, a_{n, n}, a_{12}, \ldots, a_{n-1, n}\right) \in \mathbb{P}_{f^{\prime}}^{N-1} \cap \overline{\mathbb{K}}(n),
$$

тог да имеем

1) $a_{q+1, q+1}=0, a_{q+2, q+2}=0, \ldots, a_{n, n}=0$ (иначе, по крайней мере, одна из этих величин отрицательна, в то время как форма $g(\bar{x})$ неотрищательно определенная);

2) $a_{q+1, q+2}=0, a_{q+1, q+3}=0, \ldots, a_{n-1, n}=0$;

3) $a_{i, j}=0$ при $i=1,2, \ldots, q$ и $j=q+1, q+2, \ldots, q$ (иначе, снова получаем противоречие с неотрицательностью формы $g(\bar{x}))$.

Таким образом, форма $g(\bar{x})$ - неотрицательно определенная форма только от переменных $x_{1}, x_{2}, \ldots, x_{q}$, причем, как легко видеть, произвольная.

СлЕДСТВИЕ. Для любой грани конуса $\overline{\mathbb{K}}(n)$, не совпадающей с его верииной, найдется такое $q>0$, что эта грань аффинно әквивалентна конусу $\overline{\mathbb{K}}(q)$. 
ЛЕмма 2. Пусть равенство

$$
f(\bar{x})=\sum_{i, j=1}^{n} a_{i j} x_{i} x_{j}=0
$$

определяет q-мерную вполне рациональную плоскость $\mathscr{P}^{q} \subset \mathbf{E}^{n}$, тогда множество точек Вороного, лежсащих в плоскости $\mathbb{P}_{f}^{N-1}$ с уравнением (4), аффинно әквивалентно мнохеству вершин полиэдра Вороного $\Pi(q) \subset \mathbf{E}(q)$.

ДокАЗАТЕЛьСтво. Возьмем базис пересечения $\mathscr{P} q \cap \mathbb{Z}^{n}$ и дополним его до базиса всей решетки $\mathbb{Z}^{n}$. Произведем такое линейное преобразование пространства $\mathbf{E}^{n}$, переводящее полученный базис решетки $\mathbb{Z}^{n}$ в стандартньй ее базис, чтобы плоскость $\mathscr{P} q$ перешла в плоскость $\mathbf{E}^{q}$. Это целочисленное линейное преобразование повлечет за собой (см. [1]) такое аффинное преобразование пространства $\mathbb{E}(n)$, котороепереведет плоскость (5) в плоскость $\mathbb{E}(q)$, полиэдр Вороного в себя, а точки Вороного, лежащие в (5), взаимно однозначно переведет в вершины полиэдра Вороного П $(q)$.

ЛЕмма 3. Пусть равенство (5) определяет $p$-мерную плоскость $\mathscr{P}^{p} \subset \mathbf{E}^{n}$, тогда мнохество точек Вороного, лехсащих в плоскости (4), совпадает с мнохеством точек Вороного, определенных максимальной вполне рачиональной плоскостью $\mathscr{P}_{*}^{q}$, содержащейся в плоскости $\mathscr{P}^{p}$.

ДокАЗАТЕльСтво. Случай, когда плоскость $\mathscr{P}^{p} \subset \mathbf{E}^{n}$ вполне рациональная, разобран в лемме 2. Пусть плоскость $\mathscr{P}^{p}$ не является ни вполне рациональной, ни вполне иррациональной. Заметим, что плоскость $\mathscr{P}_{*}^{q}$ однозначно определена плоскостью $\mathscr{P}{ }^{p}$ что ее можно без уменьшения общности считать плоскостью $\mathbf{E}^{q}$. Выберем теперь произвольную точку Вороного с нижними координатами, например, равными

$$
q_{1}, q_{2}, \ldots, q_{n}
$$

Если $q_{q+1}^{2}+q_{q+2}^{2}+\cdots+q_{n}^{2}=0$, то наша точка определена точкой из $\mathbf{E}^{q}$. Если же $q_{q+1}^{2}+q_{q+2}^{2}+\cdots+q_{n}^{2} \neq 0$, то подстановка строки (6) в “левую часть" уравнения плоскости $\mathscr{P}^{p}$ не может дать 0 , так как все целые точки этой плоскости находятся в плоскости $\mathbf{E}^{q}$. Следовательно, подстановка строки (6) в левую часть уравнения (5) дает число, большее нуля.

Случай вполне иррациональной плоскости уже очевиден.

ТЕОремА 1. Для каждой бесконечной грани незамкнутого совершенного полиэдра Вороного $\Pi^{-}(n)$ найдется такое $q \leqslant n$, что эта грань есть аффинный образ незамкнутого совершенного полиәдра Вороного $\Pi^{-}(q)$.

ДокАЗАТЕЛЬСТво. Случай, когда опорная плоскость к совершенному полиэдру $\Pi^{-}(n)$ вполне рациональна, разобран в лемме 2 , все другие - в лемме 3.

$6^{\circ}$. Прежде, чем перейти к основному результату, докажем несколько утверждений различного характера.

Обозначим через $\mathscr{K} \subset \mathbf{E}^{d}$ бесконечньй (не вырождающийся в полупространство) замкнутый телесньй круговой конус с вершиной в точке 0. Через $\mathscr{K}^{\prime}$ обозначим конус, который центрально симметричен конусу $\mathscr{K}$ относительно точки 0. Пусть конус $\mathscr{K}$ (или 
$\left.\mathscr{K}^{\prime}\right)$ так параллельно перенесен, что его вершина попала в точку $x$, тогда результат переноса обозначим через $\mathscr{K}_{x}$ (соответственно через $\mathscr{K}_{x}^{\prime}$ ).

Всюду дальше символом + обозначена операция векторного сложсения (сум.ма Минковского). Без потери общности мы можем и будем считать, что точка, относительно которой берется сумма Минковского, - это точка 0.

Лемма 4. Пусть $a, b, c \in \mathscr{K}$, пусть также $a+b \in \mathscr{K}_{c}^{\prime}$. Тогда a $u b \in \mathscr{K}_{c}^{\prime}$.

ДокАЗАтЕльство. Предположим сначала, что $a+b=c$. Тогда точки $\mathbf{0}, a, b$ и $c$ лежат в одной плоскости, скажем, $\mathscr{P}^{2}$, и образуют параллелограмм $\mathbf{0}, a, c, b$. Пересечение $\mathscr{P}^{2} \cap \mathscr{K} \cap \mathscr{K}_{c}^{\prime}$ также является параллелограммом с парой противоположных вершин $c$ и 0 (см. рис. 1). Очевидно, что если одна из вершин $a$ или $b$ лежит вне угла $\mathscr{P}^{2} \cap \mathscr{K}_{c}^{\prime}$, то другая лежит вне угла $\mathscr{P}^{2} \cap \mathscr{K}$. Очевидно также, что это равносильно утверждению леммы в рассматриваемом случае.

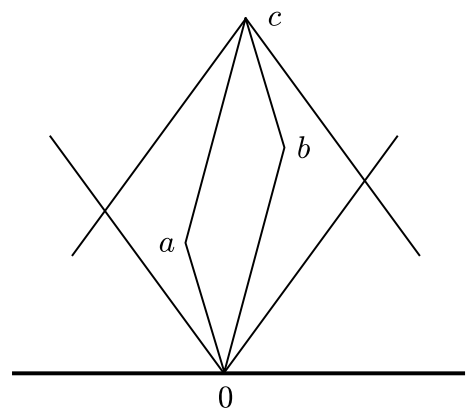

Рис. 1.

Пусть теперь $a+b=x \in \mathscr{K}_{c}^{\prime}$. Имеем $\mathscr{K}_{x}^{\prime} \subset \mathscr{K}_{c}^{\prime}$, и все следует из предыдущего случая. Лемма 5. Для любых двух множеств $A$ и $B \subset \mathscr{K}$ имеет место равенство

$$
\operatorname{clos}(A+B)=\operatorname{clos} A+\operatorname{clos} B
$$

ДокАЗАТЕЛЬСтво. 1) Пусть $x \in \operatorname{clos}(A+B)$. Выберем такую точку $c$, чтобы точка $x$ вместе с некоторой своей окрестностью $О x$ (относительно конуса $\mathscr{K}$ ) принадлежала конусу $\mathscr{K}_{c}^{\prime}$. В сколь угодно малой окрестности точки $x$ найдутся точки вида $x_{i}=a_{i}+$ $b_{i}$, где $a_{i} \in A \subset \operatorname{clos} A$ и $b_{i} \in B \subset \operatorname{clos} B$. Мы можем считать, что точки $x_{1}, x_{2}, \ldots$ образуют последовательность, сходящуюся к точке $x$ и что $x_{1}, x_{2}, \ldots \in \mathbf{O} x \subset \mathscr{K}_{c}^{\prime}$. В силу леммы 4 все точки вида $a_{i}$ и $b_{i}$ лежат в пересечении $\mathscr{K} \cap \mathscr{K}_{c}^{\prime}$. Следовательно, быть может, дважды переходя к подпоследовательности, мы можем считать, что последовательности $a_{1}, a_{2}, \ldots$ и $b_{1}, b_{2}, \ldots$ сходятся соответственно к некоторым точкам $a \in \operatorname{clos} A \cap \mathscr{K} \cap \mathscr{K}_{c}^{\prime}$ и $b \in \operatorname{clos} B \cap \mathscr{K} \cap \mathscr{K}_{c}^{\prime}$. Очевидно, что $x=a+b$, т.е.

$$
\operatorname{clos}(A+B) \subset \operatorname{clos} A+\operatorname{clos} B
$$

2) Пусть $x \in \operatorname{clos} A+\cos B$. Выберем произвольно малое $\varepsilon>0$ и через $\mathbf{O}_{\varepsilon} x \subset \mathbf{E}^{d}$ обозначим сферическую $\varepsilon$-окрестность точки $x \in \mathbf{E}^{d}$. 
Тогда в $(\varepsilon / 3)$-окрестности точки $x$ найдется точка вида $a^{\prime}+b^{\prime}$, где $a^{\prime} \in \operatorname{clos} A$ и $b^{\prime} \in \operatorname{clos} B$. Выберем точки $a \in \mathbf{O}_{\varepsilon / 3} x \cap A$ и $b \in \mathbf{O}_{\varepsilon / 3} x \cap B$, очевидно, что $a+b \in \mathbf{O}_{\varepsilon} x$. Последнее ввиду произвольной малости $\varepsilon$ эквивалентно включению

$$
\operatorname{clos} A+\operatorname{clos} B \subset \operatorname{clos}(A+B)
$$

Во всем дальнейшем мы будем считать, что

a) начало $\mathbf{0}$ конуса $\overline{\mathbb{K}}(n)$ совпадает с началом, уже $N$-мерного, конуса $\mathscr{K}$;

b) $\overline{\mathbb{K}}(n) \backslash \mathbf{0} \subset \mathscr{K}$;

c) начало векторного суммирования есть точка $\mathbf{0 .}$

ЛЕммА 6. При каждом $n \geqslant 1$ имеют место равенства

$$
(\mathbb{K}(n) \cup \mathbf{0}) \oplus \Pi^{-}(n)=\Pi^{-}(n) \quad u \quad \overline{\mathbb{K}}(n) \oplus \Pi^{+}(n)=\Pi^{+}(n) .
$$

ДОКАЗАТЕЛЬСТво. Очевидно, что

$$
\Pi^{-}(n) \subset \Pi^{-}(n) \oplus(\mathbf{0} \cup \mathbb{K}(n))
$$

Покажем противоположное включение. Каждая точка $\varphi \in \Pi^{-}(n)$ имеет вид

$$
\varphi=\sum_{1}^{N-1} \alpha_{i} l_{i}^{2}
$$

где $l_{i}$ - линейные целочисленные ненулевые формы, $\alpha_{i} \geqslant 0$ и $\sum_{1}^{N-1} \alpha_{i} \geqslant 1$. Каждая точка $\psi \in \mathbb{K}(n)$ имеет вид, см. [2, 1.1$]$ и $[1, \S 11], \varphi=\sum_{1}^{N-1} \beta_{i} l_{i}^{2}$, где $l_{i}$ - линейные целочисленные ненулевые формы, $\beta_{i} \geqslant 0$ и $\sum_{1}^{N-1} \beta_{i} \geqslant 1$.

Точка

$$
\frac{\varphi+\psi}{\sum_{1}^{N-1} \alpha_{i}+\sum_{1}^{N-1} \beta_{i}}
$$

является точкой многогранника, натянутого на вершины совершенного полиэдра $\Pi^{-}(n)$, т.е.

$$
\frac{\varphi+\psi}{\sum_{1}^{N-1} \alpha_{i}+\sum_{1}^{N-1} \beta_{i}} \subset \Pi^{-}(n) .
$$

Поскольку $\sum_{1}^{N-1} \alpha_{i}+\sum_{1}^{N-1} \beta_{i} \geqslant 1$, а для любых $g \in \Pi^{-}(n)$ и $\lambda \geqslant 1$ справедливо включение $g \lambda \in \Pi^{-}(n)$, то $\varphi+\psi \subset \Pi^{-}(n)$. Тем самым, первое из равенств леммы доказано.

Второе равенство есть прямое следствие первого и леммы 5.

ЛЕмма 7. Пусть для мнохества $\mathbb{M} \subset \overline{\mathbb{K}}(n)$ и точки $g \in \mathbb{K}(n)$ существуют плоскость $\mathbb{P}^{N-1}$, отделяюшая точку $g$ от множества $\mathbb{M}$, и такая точка $\varphi \in$ $\overline{\mathbb{K}}(n)$, что $\varphi \oplus \overline{\mathbb{K}}(n) \subset \mathbb{M}$.

Тогда существует и әллиптическая плоскость, отделяющая точку $g$ от множсества $\mathbb{M}$. 
ДокАЗАтЕльство. Во-первых, отметим, что плоскость $\mathbb{P}^{N-1}$ не гиперболическая, поскольку любая гиперболическая плоскость разрезает каждую параллельную копию конуса $\overline{\mathbb{K}}(n)$ на две полномерные части. Если плоскость $\mathbb{P}^{N-1}$ эллиптическая, то лемма доказана.

Пусть плоскость $\mathbb{P}^{N-1}$ параболическая. Без потери общности мы можем считать, что она находится на положительном расстоянии от множества М. Заметим также, что, поскольку параболический “карман” заключен между двумя плоскостями, точки $g$ и $\mathbf{0}$ лежат по одну сторону от плоскости $\mathbb{P}^{N-1}$. Опустим из них перпендикуляры на плоскость $\mathbb{P}^{N-1}$ и проведем через эти перпендикуляры двумерную плоскость $\mathbb{P}^{2}$. Прямая $\mathbb{P}^{1}=\mathbb{P}^{N-1} \cap \mathbb{P}^{2}$ пересечет границу конуса $\overline{\mathbb{K}}(n)$ в некоторой точке $\varphi$. Проведем, наконец, плоскость $\mathbb{P}^{N-2} \subset \mathbb{P}^{N-1}$, касающуюся пересечения $\mathbb{P}^{N-1} \cap \overline{\mathbb{K}}(n)$ в точке $\varphi$.

Теперь для получения искомой плоскости достаточно сколь угодно мало повернуть плоскость $\mathbb{P}^{N-1}$ вокруг плоскости $\mathbb{P}^{N-2}$ в сторону точки $g$ (но не переходя ее).

Лемма 8. Пусть $\overline{\mathbb{K}}$ - грань некоторого ранга $q$ конуса $\overline{\mathbb{K}}(n)$ u $\mathbb{P}$ - плоскость размерности $Q-1=(q+1) q / 2-1$, әллиптическая относительно конуса $\overline{\mathbb{K}}$.

Тогда существует такая әллиптическая плоскость $\mathbb{P}^{N-1}$, ито $\mathbb{P}^{N-1} \cap \overline{\mathbb{K}}=\mathbb{P}$.

ДоКАЗАТЕЛЬСТво. Произведя, если нужно, соответствующее преобразование, мы можем считать, что $\overline{\mathbb{K}}=\overline{\mathbb{K}}(q)$. Тогда общее уравнение $(Q-1)$-мерной, эллиптической относительно конуса $\overline{\mathbb{K}}(q)$, плоскости, т.е. плоскости $\mathbb{P}$, имеет вид

$$
\sum_{i, j=1}^{q} a_{i j} v_{i j}=C>0
$$

г де $\sum_{i, j=1}^{q} a_{i j} x_{i} x_{j}-$ ПК $\Phi$ от первых $q$ переменных.

Теперь уже очевидно, что уравнение

$$
\sum_{i, j=1}^{q} a_{i j} v_{i j}+v_{q+1, q+1}+\cdots+x_{n n}=C
$$

задает одну из плоскостей вида $\mathbb{P}^{N-1}$, обладаюших требуемым свойством.

7. Теорема 2. Для каждой бесконечной грани $\mathbb{G}$ замкнутого совершенного полиәдра Вороного $\Pi^{+}(n)$ найдутся такие $r \leq q \leq n$, что грань $\mathbb{G}$ есть аффинньй образ векторной суммы конуса $\overline{\mathbb{K}}(q)$ и содержсащегося в нем замкнутого совершенного полиэдра Вороного $\Pi^{+}(r)$.

ДокАЗАТЕльСтво. Пусть грань $\mathbb{G}$ определена плоскостью $\mathbb{P}_{f}^{N-1}$, заданной уравнением (4) при $\operatorname{rg} f(\bar{x})=p$, т.е.

$$
\mathbb{G}=\Pi^{+}(n) \cap \mathbb{P}_{f}^{N-1}
$$

Отметим, что пересечение $\overline{\mathbb{K}}$ нашей плоскости с конусом $\overline{\mathbb{K}}(n)$ согласно лемме 1 афффинно эквивалентно конусу $\overline{\mathbb{K}}(q)$, а также, что $\mathbb{G} \subset \overline{\mathbb{K}}$.

Без уменьшения общности мы можем считать, что максимальная вполне рациональная плоскость, содержащаяся в плоскости $\mathscr{P} q$, определенной уравнением (5), - это плоскость $\mathbf{E}^{r}$. Следовательно, $\Pi^{+}(r) \subset \mathbb{G}$.

а) Докажем включение $\overline{\mathbb{K}} \oplus \Pi^{+}(r) \subset \mathbb{G}$. 
Согласно лемме 6 имеем, что

$$
\overline{\mathbb{K}}(n) \oplus \Pi^{+}(n)=\Pi^{+}(n)
$$

Отсюда, очевидно, следует равенство

$$
\left[\overline{\mathbb{K}}(n) \oplus \Pi^{+}(n)\right] \cap \mathbb{P}_{f}^{N-1}=\Pi^{+}(n) \cap \mathbb{P}_{f}^{N-1} .
$$

Поскольку все множества, встречающиеся в этой формуле, лежат в одном и том же замкнутом полупространстве, определенном плоскостью $\mathbb{P}_{f}^{N-1}$, мы получаем

$$
\left[\overline{\mathbb{K}}(n) \cap \mathbb{P}_{f}^{N-1}\right] \oplus\left[\Pi^{+}(n) \cap \mathbb{P}_{f}^{N-1}\right]=\Pi^{+}(n) \cap \mathbb{P}_{f}^{N-1}
$$

Отсюда, учитьвая, что $\mathbb{G}=\Pi^{+}(n) \cap \mathbb{P}_{f}^{N-1}$, что $\overline{\mathbb{K}}(n) \cap \mathbb{P}_{f}^{N-1}=\overline{\mathbb{K}}$ и что $\mathbb{G} \subset \overline{\mathbb{K}}$, имеем $\overline{\mathbb{K}} \oplus \mathbb{G} \subset \mathbb{G}$. И окончательно, учитьвая, что $\Pi^{+}(r) \subset \mathbb{G}$, имеем

$$
\overline{\mathbb{K}} \oplus \Pi^{+}(r) \subset \mathbb{G} .
$$

б) Докажем включение $\mathbb{G} \subset \overline{\mathbb{K}} \oplus \Pi^{+}(r)$.

Возьмем произвольную точку

$$
g \in \overline{\mathbb{K}} \backslash\left(\overline{\mathbb{K}} \oplus \Pi^{+}(r)\right)
$$

и покажем, что $g \bar{\in} \mathbb{G}$. Действительно, множество $\mathbb{M}=\overline{\mathbb{K}} \oplus \Pi^{+}(r)$ как векторная сумма двух замкнутых и выпуклых множеств, замкнуто и вьпукло. Следовательно, относительно пары $g$ и $\mathbb{M}$ вьполнены все условия леммы 7, тем самым существует эллиптическая относительно конуса $\mathbb{K}$ плоскость, отделяющая точку $g$ от множества $\mathbb{M}$. Согласно лемме 8 эта плоскость может быть продолжена до некоторой, эллиптической относительно конуса $\mathbb{K}(n)$ плоскости $\mathbb{P}^{N-1}$. Плоскость $\mathbb{P}^{N-1}$ отделяет множество Мот точки $g$ и, вообще говоря, еще от конечного числа точек Вороного. Повернув плоскость $\mathbb{P}^{N-1}$ относительно плоскости $\mathbb{P}^{N-2} \subset \mathbb{P}^{N-1}$, касательной к пересечению $\mathbb{P}^{N-1} \cap \mathbb{K}(n)$, мы найдем плоскость, отделяющую точку $g$ от множества $\mathbb{M}$ и от всех точек Вороного, лежащих вне него. Таким образом, $g \bar{\in} \mathbb{G}$, что эквивалентно утверждаемому включению.

\section{СПИСОК ЦИТИРОВАННОЙ ЛИТЕРАТУРЫ}

[1] Рышков С. С., Барановский Е. П. Классические методы теории решетчатых упаковок // УМН. 1979. Т. 34. № 4 (208). С. 3-63.

[2] Вороной Г.Ф. Исследования о примитивных паралеллоэдрах // Собр. соч. Т. 2. Киев: Изд-во АН УССР, 1952. С. 239-368. (Оригинал в J. Reine Angew. Math., 1908, V. 134, P. 198-287, 1909, V. 136, P. 67-179). 